Relations industrielles

Industrial Relations

\title{
Sainsaulieu, Ivan, La contestation pragmatique dans le syndicalisme autonome: la question du modèle SUD-PTT
}

\section{Patrick Rozenblatt}

Volume 55, numéro 3, 2000

URI : https://id.erudit.org/iderudit/051342ar

DOI : https://doi.org/10.7202/051342ar

Aller au sommaire du numéro

Éditeur(s)

Département des relations industrielles de l'Université Laval

ISSN

0034-379X (imprimé)

1703-8138 (numérique)

Découvrir la revue

Citer ce compte rendu

Rozenblatt, P. (2000). Compte rendu de [Sainsaulieu, Ivan, La contestation pragmatique dans le syndicalisme autonome : la question du modèle SUD-PTT]. Relations industrielles / Industrial Relations, 55(3), 551-553.

https://doi.org/10.7202/051342ar

Tous droits réservés @ C Département des relations industrielles de l'Université Laval, 2000
Ce document est protégé par la loi sur le droit d'auteur. L'utilisation des services d'Érudit (y compris la reproduction) est assujettie à sa politique d'utilisation que vous pouvez consulter en ligne.

https://apropos.erudit.org/fr/usagers/politique-dutilisation/ 
recognized them for what they were and would stay - a party dedicated to reform but not to socialism. But Lenihan knew there was no other option and strove to implement political and trade union reforms that changed the landscape in a very positive way for workers across Canada.

But the challenge for the labour movement now is to decide whether the vision for reform rests within the existing labour and political alliances or within a new breakaway labour central and an alternative political strategy espoused by an industrial union with aspirations to increase its public sector membership.

What would Patrick Lenihan think? He certainly would be saddened by a possible dissolution of the labour central and the wedge that is being driven between public sector workers. But one thing that is clear from this book is that you have to be close to the action in order to have a vision for the future.

Editor Gil Levine has done a great job of capturing Lenihan's life in his own words so that we can be as close as possible to being in Lenihan's shoes as he made decisions about his own political and trade union life. Those decisions helped shape our world today. It may take another book fifty years from now to detail the inner workings of the labour movement in 2000 in order to assess whether current machinations within the labour movement are vision or folly.

Stan Marshall Canadian Union of Public Employees

\section{La contestation pragmatique dans le syndicalisme autonome : la question du modèle SUD-PTT}

par Ivan SaInSAUlieu, Paris : L'Harmattan, Collection Logiques Sociales, 1999, 287 p., ISBN 2-7384-7616-3.

Pendant longtemps l'approche de la question syndicale en France s'est résumée au traitement des orientations stratégiques des cinq organisations confédérées décrétées représentatives par le pouvoir politique - Confédération générale du travail (CGT), Confédération française démocratique du travail (CFDT), Force ouvrière (FO), Confédération française des travailleurs chrétiens (CFTC), Confédération générale des cadres (CGC) - , auxquelles un regard déjà plus spécialisé ajoutait la Fédération de l'éducation nationale (FEN). Or, ces quinze dernières années, dans le cadre plus général d'une crise de la représentativité politique et sociale, toutes ces organisations ont été de plus en plus contestées dans leur droit à conserver l'exclusivité pour parler, agir et négocier au nom de tous les travailleurs salariés.

De l'émergence des coordinations comme forme d'organisation de la lutte sociale (1986) au développement d'un mouvement associatif diversifié autour de questions de société (1994), plus ou moins négligées par l'action syndicale confédérée comme le logement, le chômage et l'immigration, les espaces sont nombreux pour l'expression d'un syndicalisme autonome lui-même en recherche d'identité. C'est à l'exploration de cette réalité toujours très actuelle que nous convie l'ouvrage politologique d'Ivan Sainsaulieu. Il est essentiel de préciser le parti pris disciplinaire de l'auteur car celui-ci détermine une problématique et un cheminement spécifique autour de l'enjeu étudié. En effet, dès son introduction, l'auteur situe son analyse au cœur des catégories de pensée et des outils du politologue qui, face à des formes institutionnelles, cherche à les qualifier à travers le prisme binaire de l'étude de plusieurs dyades, telles réforme-révolution, confédéréautonome, politique-apolitique. Dans ce cadre, l'objet du livre vise à établir la nature du nouveau syndicalisme 
autonome afin de savoir si ceux qui y militent « veulent simplement revivifier le syndicalisme comme contre pouvoir, ou en faire un vecteur de la transformation sociale, ou encore appuyer des acteurs politiques pour qu'ils jouent un rôle transformateur?» (p. 6). L'auteur nous conduit à travers trois chapitres vers sa réponse.

Dans le premier chapitre, il se livre à une large présentation du syndicalisme autonome (ou non confédéré) qui puise ses principales racines dans le refus de la division de 1949, entre la CGT et FO, contraire, selon lui, aux principes essentiels de la Charte d'Amiens. Il existe principalement au sein du secteur public où loin d'être un phénomène marginal il représente, toutes tendances confondues, $48 \%$ des suffrages aux élections professionnelles. Suite aux multiples recompositions entreprises depuis le début des années quatre-vingt, le syndicalisme autonome se compose de trois grands pôles: l'Union nationale des syndicats autonomes (UNSA) reconnue depuis peu à l'égale d'une confédération avec $18,06 \%$; la Fédération syndicale unifiée (FSU) désormais majoritaire dans l'éducation nationale avec $14,71 \%$; les divers, dans lesquels figurent Solidaireunitaire-démocratique aux postes et télécommunications (SUD-PTT) et le Syndicat national unifié des impôts (SNUI) avec $15 \%$, ces deux structures animant une fédération dénommée G-10 (Groupe des dix). C'est au dernier pôle que l'auteur s'intéresse en particulier à travers l'histoire contrastée des origines syndicales de ses diverses composantes et des luttes menées, notamment celles des années quatre-vingt-dix, à travers la rencontre avec un nouveau type de mouvement associatif.

Dans le deuxième chapitre, Ivan Sainsaulieu cherche à expliquer le dynamisme de SUD-PTT, créé en 1989 par des militants exclus de la CFDT suite au déroulement d'un conflit sectoriel, et à qualifier l'engagement du syndicat entre contestation et pragmatisme. Il examine pourquoi «après seulement huit ans d'existence, il obtient une moyenne de 21,5\% des voix aux élections professionnelles, se plaçant en $4^{\mathrm{e}}$ position à $\mathrm{La}$

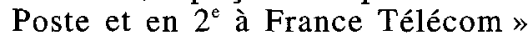
(p. 102). On apprend ainsi à découvrir les multiples visages de ce syndicalisme « de la base », syndicalisme de « francs tireurs » et « de pionniers » animé par d'anciens militants révolutionnaires de 1968. Il devrait son succès à l'ambiguité fondamentale que recèlerait son projet syndical : «professionnel et moral plus que politique » (p. 109), « sachant allier la connaissance des dossiers à la proximité du terrain, le recours à la grève et l'utilisation des failles juridiques du système » (p. 132). C'est de cette nature que découle, selon l'auteur, l'aptitude du syndicat à s'attaquer aux problèmes de société et sa capacité à nouer des relations larges avec d'autres formes sociales contestatrices. Il confirme la valeur de son hypothèse en commentant les résultats détaillés d'une enquête par questionnaire postal qu'il a initié auprès des adhérents. En se référant aux diverses générations qui construisent depuis dix ans la fédération dans un contexte de crise du service public, il conclut au mariage réussi entre les dirigeantsfondateurs, soixante-huitard politiquement formés, et les nouveaux militants emprunts de pragmatisme dans leur révolte.

Dans le troisième chapitre, Ivan Sainseaulieu développe le fond de sa thèse sur le double sens de la contestation pragmatique. Pour tenir compte de la spécificité française, il choisit dans son approche historique d'envisager l'évolution de ce processus à travers une lecture dont la ligne de partage s'avère être le puissant mouvement social de 1995. Tout en insistant sur l'originalité du militantisme déployé, notamment au contact du mouvement associatif qui agit sur divers aspects de la société civile (DAL pour le droit au logement, Act Up dans la lutte contre le sida, AC ! dans le cadre du mouvement de lutte contre le 
chômage...), il soutient qu'avant 1995 domine dans le syndicalisme autonome un pragmatisme de type local caractérisant alors les syndicalistes qui y participent comme « réformistes par défaut, au sens où ils s'inscrivent, même sans le dire explicitement, dans le cadre des institutions sociales et politiques existantes, qu'ils entendent améliorer en approfondissant leur caractère démocratique " (p. 183). Par contre, la configuration ouverte par le «Tous Ensemble» de la contestation sociale de type global de 1995 (p. 209), engendre une évolution radicale qui atteint SUD-PTT et plus largement les syndicats qui se créent alors à son image. Dès lors, «avec le maintien d'un lien fort entre des sujets aussi différents que la question salariale, le chômage, la question des femmes, des immigrés, de l'écologie, et, localement, de l'antinucléaire. Plutôt qu'un lieu d'éclatement des liens, le pragmatisme inventif de SUD constitue plutôt le point de départ d'un renouveau syndicalpolitique » (p. 255). Déjà aiguillon et novateur, le syndicalisme autonome se voit, en conclusion, invité par Ivan Sainsaulieu à « chercher des idées, à théoriser et à se saisir des opportunités pour créer des mobilisations qui font date » (p. 273).

Cet ouvrage apporte assurément sa contribution à une meilleure connaissance de la société française en explorant un espace où les tensions et les contradictions n'ont cessé de croître ces vingt dernières années. Tout juste peuton regretter, mais il s' agit certainement ici d'un biais disciplinaire, qu'à la recherche d'une caractérisation de ce syndicalisme autonome, l'auteur ne se soit pas engagé dans une réflexion critique sur les catégories mêmes de la représentation politique. Il aurait ainsi pu établir que la crise de la représentation spéculaire pousse à l'articulation d'une complémentarité entre démocratie représentative et démocratie directe, qu'elle explique la confrontation entre formes fédérées et confédérées, qu'elle interdit désormais aux syndicats, comme aux partis d'ailleurs, de se comporter comme une avant-garde dépositaire d'une « juste » orientation de classe. Dès lors, en soutenant que l'action syndicale ne se réduit pas à la consultation des salariés ou des usagers-citoyens, mais qu'il inclut leur intervention et leur mobilisation dans les modes de décisions, on peut défendre que les SUD et les autres syndicats du G10 participent largement à l'institution imaginaire de la société à travers l'apport fournit au processus de démocratisation des rapports sociaux. Pour l'essentiel, ils stimulent un élément émergent de cette recherche de citoyenneté généralisée qui travaille avec des catégories originales nos sociétés au quotidien.

Patrick Rozenblati Travail et Mobilités-CNRS Université Paris 10

\section{Contingent Work: American Employment Relations in Transition}

edited by Kathleen BARKER and Kathleen CHRISTENSEN, Ithaca, NY: ILR Press, 1998, 350 p., ISBN 0-8014-8405-7 (pbk.: alk. paper) and ISBN 08014-3369-X (cloth: alk. paper).

This is one of the recent additions to books focusing on transitions taking place in American employment relations. The book focuses on contingent work of temporary, self-employed contract, or involuntary part-time work. All chapters, except one, are on the U.S. labour market and the experiences of contingent workers there. Although the focus is on the U.S., there is much to learn from its experiences, particularly for those who are newcomers to this field. One of the important contributions of the book is to bring together thinkers from diverse fields such as anthropology, sociology, social psychology, 